\title{
The Study of Active and Passive Transducers
}

\begin{abstract}
S.Abhishek
Abstract: This paper illustrates the working of active and passive transducers, their characteristics and it lights up the theoretical study with its application. This thesis focuses the real world use of active and passive transducers with their major types of classifications.
\end{abstract}

Keywords: Sensing element, transductiom element, Thermocouple, LDR.

\section{INTRODUCTION}

A transducer is a device which converts a non-electrical Following are the items that can be classified under this physical quantity (i.e. temperature, sound or light) into an characteristics : electrical signal (i.e. voltage, current, capacity...)

a) Range or Span

In most of the electrical systems, the input signal will not be an electrical signal This will have to be converted into its corresponding electrical signal for its value to be measured using electrical methods.

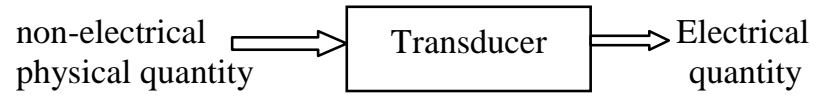

It defines the maximum and minimum values of the inputs or the outputs for which the instrument is recommended to use.

\section{b)Sensitivity}

It can be defined as the ratio of the incremental output and the incremental input. While defining the sensitivity, we assume Elekgticicaltgeantititput-output characteristic of the instrument is app linear in that range. Again Sensitivity of a system may vary with the temperature or other external factors this is known as Sensitivity drift

\section{c)Linearity}

Linearity is the deviation of the plotted transducer output from a straight line for successive equal increments of the input and it $\mathrm{s}$ often described as the percentage of the max or full scale output. It is actually the measure of non linearity of the system

The transducer has basically two main components :

\section{Sensing Element}

The physical quantity or its rate of change is sensed and responded to by this part of the transducer

\section{Transduction Element}

The output of the sensing element is passed on to the transduction element. This element is responsible for converting the non-electrical signal into its proportional electrical signal.

There are certain cases when the transduction element itself performs both the action of transduction and sensing. The best example of such a type transducer is a thermocouple.

\section{Input :}

Some examples of input are : Resistance ,capacitance , inductance, heat

\section{Output :}

Some examples of output are : voltage , force , displacement, pressure ,current.

\section{CHARACTERISTICS OF TRANSDUCER} A System generally has two characteristics namely a) Static characteristics

b)Dynamic characteristics

\section{A) STACTIC CHARCTERSTICS}

Static characteristics refer to the characteristics of a system whose input is held either constant or varying very slowly.

\section{d) Resolution}

It is the smallest difference between the measured that can be discriminated. Ex : last stable figures in the digital display .

\section{e)Accuracy}

Accuracy indicates the closeness of the measured value with the actual or true value, and is expressed in the form of the maximum error $=($ measured value true value) as a percentage of full scale reading. It also defines that the errors will not exceed when the instrument is used/operated under stated operating conditions.

\section{f) Repeatability}

The repeatability is the agreement among a number of consecutive measurements of the output for the same value of the input - under the same operating conditions and when approached from the same direction.

\section{g) Reproducibility}

The reproducibility is the agreement among a number of consecutive measurements of the output for the same value of the input - under the same operating conditions and when approached from the either direction.

\section{h) Hysterics}

The effect in which the output differs for the same value of input if the input is applied in the increase direction vs decrease direction, is called hysteresis. 


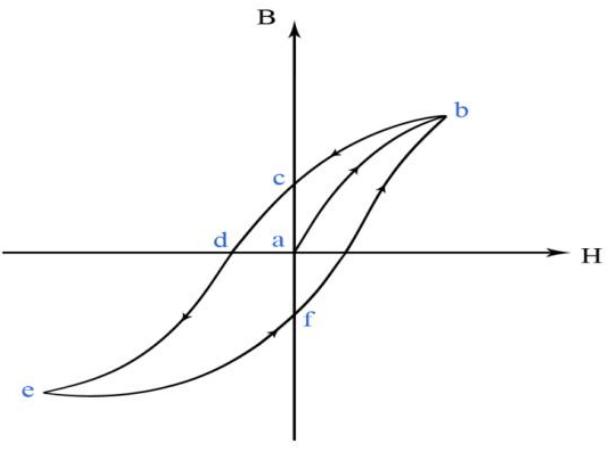

c- Maximum Output hysteresis

f- Maximum input hysteresis

\section{B) DYNAMIC CHARCTERISTICS :}

Dynamic characteristics refers to the characteristics of a system whose input varies with the time. Following are the items that can be classified under this characteristics:

\section{Response time}

It is the time interval between a change in the measured quantity and the time an instrument reads a new equilibrium value, and its often defined in terms of three characteristic times:

\section{a)Dead time}

Time during which a new signal or variation in a signal cannot be detected due to some physical characteristic of the system or the transducer.

\section{b)Rise time :}

The time taken by the instrument to respond to a step change in a measured quantity, often defined as the time taken to change from $10 \%$ to $90 \%$ of the final value.

\section{c)Settling time}

The time required for an instrument to attain a stable reading within a stated percentage of its equilibrium or it can also be defined as the time taken to attain the response within $\square \square 2 \%$ of the steady state value .

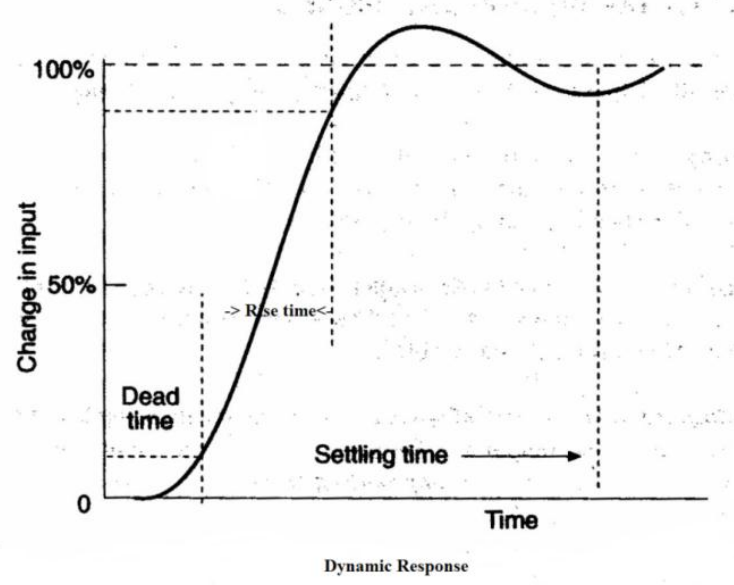

III. ACTIVE TRANSDUCERS

Active transducers are those transducers that don't require any power source for their operation. They work on energy conversion principle i.e produces an electrical signal proportional to the input ( physical quantity ).

Some of the active transducers are

\section{1) THERMOCOUPLE}

Thermocouples are the devices that are extensively used for measurement of temperature in industrial situations.

\section{PRINCIPLE OF OPERATION :}

Thermocouples works on 3 effects namely

i)SEE-BACK EFFECT:- When two conductors made from dissimilar metals are connected together forming 2 common junctions, a net thermal emf is produced at the two junctions. The amount of emf generated is different for different combination of metals.

ii) PELTIER EFFECT :- When two conductors made from dissimilar metals are connected together forming 2 common junctions, a net thermal emf is produced within the circuit due to the different temperatures of two junctions of the circuit

iii) THOMSON EFFECT :- When two conductors made from dissimilar metals are connected together forming 2 common junctions, potential exist within the circuit due to the temperature gradient along the entire length of the conductor within the circuit.

In most cases the emf suggested by the Thomson effect is very small and it can be neglected by proper selection of metals.

\section{WORKING}

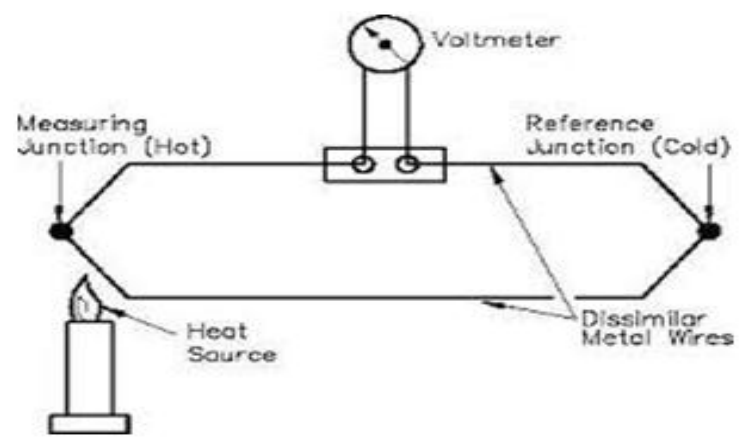

The thermocouple consist of two dissimilar metals. These are joined to form two junctions namely Hot and Cold junctions which are maintained at two different temperatures .Since two junctions are maintained at different temperatures the peltier emf is generated within the the circuit and is the function of the temperatures of two junctions.

If the temperature of the both junctions are equal and opposite emf will be generated at both the junctions and net current flowing the junction is zero .so the junction should be maintained at different temperatures so that the net emf is not zero and so there will current flowing through the circuit. The total emf developed depends on the metals used as well as the temperature of the two junctions the total emf can be measured by using suitable devices like voltmeter etc.

Now the temperature of the reference junction is known ,while the temperature of the measuring junction is unknown. The output thus obtained is calibrated directly against the unknown temperature. Thus voltage or current obtained from the circuit gives the value of unknown temperature directly. 
ADVANTAGES OF THERMOCOUPLE

i) They are rugged and readings are always consistent

ii) They can measure over a wide range of temperature upto $2600^{\circ} \mathrm{C}$

iii) Their characteristics are almost linear with an accuracy of about $\square \square 0.05 \%$.

iv)They have high resolution.

\section{2)PIEZOELECTRIC TRANSDUCER}

Piezoelectric transducer/sensor is a active transducer and it does not need external power supply as it is self generating. It is the devices which uses piezoelectric effect to measure the changes in pressure, temperature, acceleration, strain by converting them to electrical pulse.

\section{PRINCIPLE OF OPERATION}

Piezoelectric effect - There are certain materials that generates a electric potential or voltage when a mechanical strain is applied to them, they tend to change the dimensions along plane. This effect is called piezoelectric effect . Piezoelectric transducers uses this principle for measurement of pressure, temperature etc.

\section{WORKING}

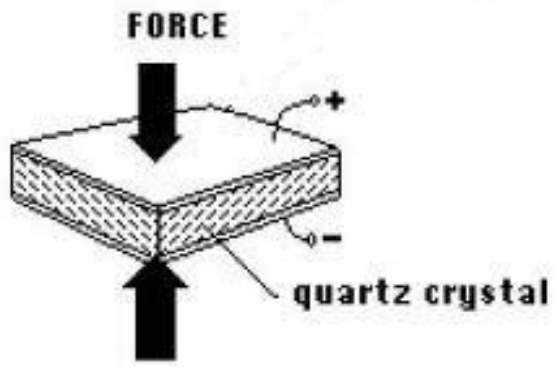

When a mechanical stress or forces are applied to some materials along certain planes, they produce electric voltage. This voltage can be measured simply by the voltage measuring instruments. This voltage measured gives the stress or force applied. Some of the materials that exhibit piezoelectric effect are quartz, Rochelle salt, polarized barium titanate, Ordinary sugar etc...

\section{ADVANTAGES OF PIEZOELECTRIC SENSOR \\ 1)High frequency response \\ 2)High transient response \\ 3) Rugged in construction \\ 4) They are small in size}

\section{PASSIVE TRANSDUCERS}

Transducers which require external power supply for their operation is called passive transducers. They produce an output signal in the form of some variation in resistance, capacitance or any other electrical parameter, which than has to be converted to an equivalent current or voltage signal.

\section{1)LIGHT DEPENDENT RESISTOR (LDR)}

A Light Dependent Resistor (LDR) or a photo resistor is a device whose resistivity is a function of the incident electromagnetic radiation. Hence, they are light sensitive devices. They are also called as photo conductors, photo conductive cells or simply photocells. They are made up of semiconductor materials having high resistance. There are many different symbols used to indicate a LDR, one of the most commonly used symbol is shown in the figure below. The arrow indicates light falling on it.

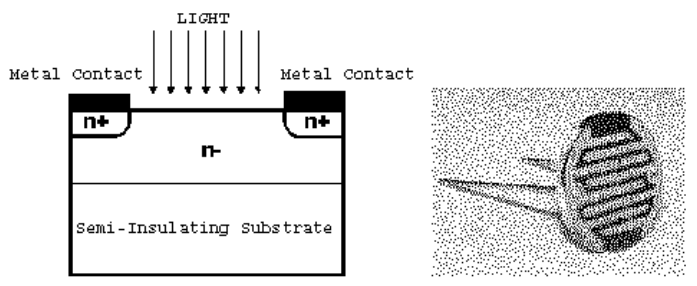

The structure of a light dependent resistor consists of a light sensitive material which is deposited on an insulating substrate such as ceramic. The material is deposited in zigzag pattern in order to obtain the desired resistance \& power rating. This zigzag area separates the metal deposited areas into two regions. Then the ohmic contacts are made on the either sides of the area. The resistances of these contacts should be as less as possible to make sure that the resistance mainly changes due to the effect of light only. Materials normally used are cadmium sulphide, cadmium selenide, indium antimonide and cadmium sulphonide. The use of lead and cadmium is avoided as they are harmful to the environment.

\section{PRINCIPLE OF WORKING}

A light dependent resistor works on the principle of photo conductivity. Photo conductivity is an optical phenomenon in which the materials conductivity (Hence resistivity) reduces when light is absorbed by the material. When light falls i.e. when the photons fall on the device, the electrons in the valence band of the semiconductor material are excited to the conduction band. These photons in the incident light should have energy greater than the band gap of the semiconductor material to make the electrons jump from the valence band to the conduction band. Hence when light having enough energy is incident on the device more \& more electrons are excited to the conduction band which results in large number of charge carriers. The result of this process is more and more current starts flowing and hence it is said that the resistance of the device has decreased. This is the most common working principle of LDR

\section{CHARACTERSTICS OF LDR}

LDR's are light dependent devices whose resistance decreases when light falls on them and increases in the dark.

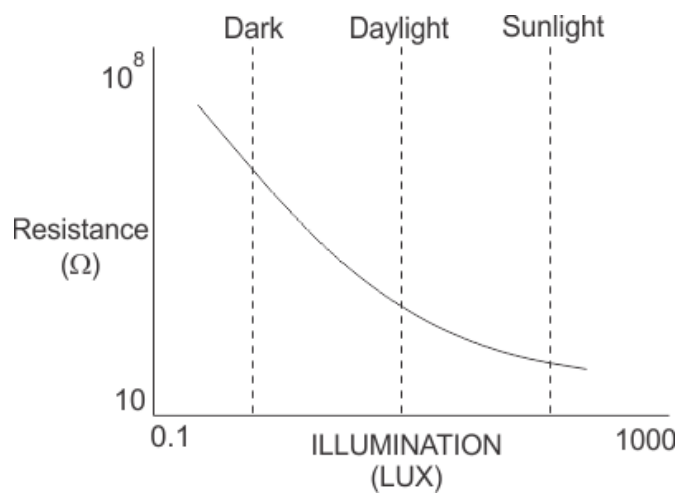


When a light dependent resistor is kept in dark, its resistance is very high. This resistance is called as dark resistance. It can be as high as $1012 \Omega$. And if the device is allowed to absorb light its resistance will decrease drastically. If a constant voltage is applied to it and intensity of light is increased the current starts increasing. Figure shows resistance vs. illumination curve for a particular LDR.

\section{2)STRAIN GUAGE}

Fundamentally, all strain gages are designed to convert mechanical motion into an electronic signal. A change in capacitance, inductance, or resistance is proportional to the strain experienced by the sensor. If a wire is held under tension, it gets slightly longer and its cross-sectional area is reduced. This changes its resistance $(\mathrm{R})$ in proportion to the strain sensitivity $(\mathrm{S})$ of the wire's resistance. When a strain is introduced, the strain sensitivity, which is also called the gage factor(GF), is given by:

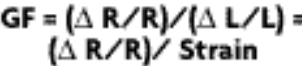

The ideal strain gage would change resistance only due to the deformations of the surface to which the sensor is attached. However, in real applications, temperature, material properties, the adhesive that bonds the gage to the surface, and the stability of the metal all affect the detected resistance. Because most materials do not have the same properties in all directions, a knowledge of the axial strain alone is insufficient for a complete analysis. Poisson, bending, and torsional strains also need to be measured. Each requires a different strain gage arrangement.

\section{PRINCIPLE OF OPERATION}

Each metal has its specific resistance. An external tensile force /(compressive force) increases/decreases the resistance by elongating/contracting it. Suppose the original resistance is $\mathrm{R}$ and a strain initiated change in resistance is $\Delta \mathrm{R}$. Then, the following relation is concluded:

$$
\frac{\Delta \mathrm{R}}{\mathrm{R}}=\mathrm{Ks} \cdot \frac{\Delta \mathrm{L}}{\mathrm{L}}=\mathrm{Ks} \cdot \mathcal{\varepsilon}
$$

where, Ks is a gauge factor, the coefficient expressing strain gauge sensitivity. General purpose strain gauges use copper-nickel or nickel-chrome alloy for the resistive element, and the gauge factor provided by these alloys are approximately 2 .

\section{WORKING}
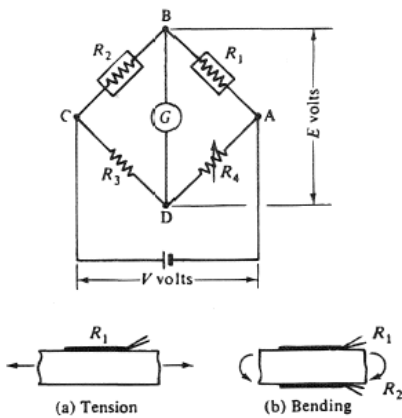

The above diagram shows the electrical construction of wheatstone bridges, with and without the application of strain.

External force applied to a ferritic material generates physical deformation and electrical resistance change of the material. In case that such material is sticked onto test specimen via electrical insulation, the material produces a change of electrical resistance corresponding to the deformation. Strain gauges consist of electrical resistance material and measure proportional strains to the resistance changes. The complete Wheatstone Bridge is excited with a stabilised DC supply and with additional conditioning electronics, can be zeroed at the null point of measurement. As stress is applied to the bonded strain gauge, a resistive changes takes place and unbalances the Wheatstone Bridge.

\section{RESULT}

Hence this paper exemplifies the in-depth study of active and passive transducers with diagrammatic way. Strongly lights up the technical aspects of transducers with perfect theoretical survey.

\section{REFERENCES}

www.electronics-tutorials.ws

www.quora.com

Book: Mechanical and Industrial Measurements by R. K. Jain, Khanna Publishers

https://www.nde-ed.org/EducationResources

www.mfg.mtu.edu

WWW.sciencedirect.com

www.signal.uu.se

www.egr.msu.edu/classes 